\title{
The Epidemiological Profile of Candidemia at an Indian Trauma Care Center
}

\section{Vibhor Tak, Purva Mathur, Prince Varghese', Jacinta Gunjiyal' ${ }^{1}$ Immaculata Xess ${ }^{2}$, Mahesh C Misra ${ }^{3}$}

Departments of Laboratory Medicine, ${ }^{1}$ Hospital Infection Control and ${ }^{3}$ Surgery, JPNA Trauma Center, ${ }^{2}$ Department of Microbiology, AllMS, New Delhi, India

Address for correspondence: Dr. Purva Mathur, E-mail: purvamathur@yahoo.co.in

\section{ABSTRACT}

Purpose: Candida spp. is a common cause of bloodstream infections. Candidemia is a potentially fatal infection that needs urgent intervention to salvage the patients. Trauma patients are relatively young individuals with very few comorbidities, and the epidemiology of candidemia is relatively unknown in this vulnerable and growing population. In this study, we report the epidemiology of candidemia in a tertiary care Trauma Center of India.

Materials and Methods: The study was conducted from January 2009 to July 2012. All patients from whose blood samples a Candida spp. was recovered were included in this study. A detailed history and follow up of the patients was done. The isolates of Candida were identified to the species level. The speciation was done by conventional methods, including morphology on Corn Meal Agar, color development on Triphenyl Tetrazolium Chloride Agar and CHROMagar, and germ tube tests. The VITEK 2 YST ID colorometric card, a fully automated identification system was also used. Antifungal susceptibility was performed using the VITEK 2 system.

Results: A total of 212 isolates of the Candida species were recovered from blood samples of 157 patients over the study period. Candida tropicalis, 82 (39\%), was the most common, followed by C. parapsilosis, $43(20 \%)$, C. albicans, 29 (14\%), C. glabrata, 24 (11\%), C. rugosa, 20 (9\%), C. hemulonii,; 6 (3\%), C. guilliermondii, 4 (2\%), C. famata, $3(1.5 \%)$, and C. Iusitaniae $1(0.5 \%)$. Out of all the candidemia patients, $68(43 \%)$ had a fatal outcome. Fluconazole and Amphotericin B resistance was seen in seven (3.3\%) and seven (3.3\%) of the isolates, respectively.

Conclusion: Candidemia is a significant cause of mortality in trauma patients in our center, with $C$. tropicalis and C. parapsilosis being the predominant pathogens. Resistance to antifungal drugs is a matter of concern. Better hospital infection control practices and good antibiotic stewardship policies could possibly help in reducing the morbidity and mortality associated with candidemia.

Key words: Antifungal resistance, blood stream infections, candidemia, trauma

\section{INTRODUCTION}

Fe ungi have emerged as major opportunistic pathogens causing human disease. Candida accounts for nearly $96 \%$ of all opportunistic mycoses and is an important cause of blood stream infections (BSI). ${ }^{[1]}$ It is currently the fourth most common nosocomial blood stream isolate

\begin{tabular}{|c|c|}
\hline \multicolumn{2}{|c|}{ Access this article online } \\
\hline 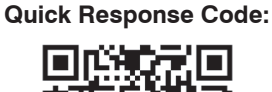 & $\begin{array}{l}\text { Website: } \\
\text { www.jlponline.org }\end{array}$ \\
\hline 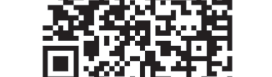 & $\begin{array}{l}\text { DOI: } \\
10.4103 / 0974-2727.141506\end{array}$ \\
\hline
\end{tabular}

in USA. ${ }^{[1,2]}$ Invasive candidiasis is recognized as a leading cause of morbidity and mortality in critically ill patients, with reported crude mortality rates due to candidemia, ranging from $30-81 \% \cdot{ }^{[3,4]}$ Candidemia is also associated with prolonged hospital stay and increased healthcare costs..$^{[5-7]}$ Delaying specific antifungal treatment significantly increases the mortality in invasive candidiasis. ${ }^{[8-10]}$ Thus, a high index of suspicion, early diagnosis, and a prompt and appropriate therapy remains the cornerstone of treatment.

The epidemiology of Candidemia is evolving. In the past, a majority of the opportunistic fungal infections due to Candida and Aspergillus were described in immunocompromised hosts and primarily cancer 
patients, but nowadays, these infections are occurring more commonly in non-immunocompromised surgical and critically ill patients. ${ }^{[1]]}$ An estimated $33-55 \%$ of all episodes of candidemia occur in Intensive Care Units (ICU). ${ }^{[12]}$

During recent decades, there is a progressive shift from a predominance of Candida albicans toward a predominance of non-albicans Candida spp., especially C. glabrata, C. parapsilosis, and C. tropicalis, in both neutropenic and non-neutropenic critically ill patients. There is growing evidence suggesting a role of the increasing use of azole agents in this epidemiological shift. In some North American and European centers reduced susceptibility to commonly used antifungal agents has also been observed..$^{[13,14]}$

Candidemia occurs due to colonization of skin and mucosal membranes by Candida and disruption in the host's natural epithelial barrier, due to burns, surgery or insertion of intravascular catheters. Candidemia can occur in a hospital setting as a primary Central Line Associated Bloodstream Infection (CLABSI) or secondary bloodstream infection. There are multiple risk factors for developing candidemia, namely, neutropenia, hematological malignancies, cancer chemotherapy, organ transplant therapy, human immunodeficiency virus infection/acquired immunodeficiency syndrome (HIV/AIDS), diabetes, and so on.

In the burns and trauma patients and other critically ill medical and surgical patients, use of central venous catheters, surgical procedures, total parenteral nutrition, admission to an Intensive Care Unit, mucosal colonization by candida and use of broad spectrum antibiotics predispose these patients to develop blood stream infections by Candida spp. ${ }^{[11]}$ On account of such a large number of cases occurring in the ICUs, the focus of candidemia studies has shifted from neutropenic patients toward critically ill non-neutropenic patients.

The critically ill trauma patients are relatively young individuals, who have been healthy previously, but trauma exposes them to microbial pathogens at the time of injury. They are often on invasive devices and broad spectrum antibiotics, with prolonged hospitalization periods, which further predisposes them to develop candidemia. Although invasive candidiasis is well studied in immunocompromised patients, its epidemiology in the growing and vulnerable 'trauma' population is relatively unknown. This study reports the profile of candidemia at a tertiary care Indian trauma center.

\section{MATERIAL AND METHODS}

The study was conducted at a tertiary care trauma center of the All India Institute of Medical Sciences, from January 2009 to July 2012. The center is 180 bedded, with two ICUs, having 32 beds totally. All patients diagnosed as having candidemia were included in the study. Candidemia was defined as the isolation of any Candida spp. from one or more blood culture samples of a patient. A detailed proforma was filled for all patients included in the study. The information included patient demography, the specific fungal pathogen isolated and its species, resistance to antifungals, and the final outcome of the patients. The clinical outcomes of all patients with candidemia were recorded, until their death/discharge from the hospital.

Candida isolates from patient's blood culture and central venous catheter tips were identified up to the species level, using both conventional and automated techniques. ${ }^{[15]}$ Conventional identification was performed by observing the morphology on the Corn Meal Agar, with color development on the Triphenyl Tetrazolium Chloride Agar, the CHROMagar (BD, Franklin Lakes, New Jersey, USA), and germ tube tests. The VITEK 2 YST ID colorometric card was used for automated identification. Antifungal susceptibility was performed using the VITEK 2 system (bioMerieux Pvt. Ltd., St. Vulbas, France). The following antifungal drugs were tested: Amphotericin-B, Fluconazole, Flucytosine, and Voriconazole. The conventional method of identification was taken as our gold standard in cases of discordance in the identification of Candida spp. by the automated VITEK 2 system and the conventional methods. If the concordance/probability of the isolate identification by VITEK 2 was less than $95 \%$ or the isolate was not identified by VITEK 2 or the isolate was identified with a low discrimination between various species, then in such cases the morphological identification of the Candida spp. was taken as accurate and reported as such. During the study period there were no changes in the microbiological laboratory techniques.

\section{RESULTS}

During these 43 months of the study period, from January 2009 to July 2012, a total of 20,240 patients were admitted to our trauma center. Six thousand eight hundred and twenty-two patients were critically injured and required intensive monitoring and life support management, and were therefore, admitted to the Intensive Care Unit. The remaining 13,418 patients were relatively more stable, but had injuries severe enough to merit their admission to 
hospital, and were subsequently admitted in our surgical wards. During the study period, a total of 212 isolates of Candida spp. were obtained from 157 patients. Candidemia developed in 102 and 55 patients admitted to the ICU and wards, respectively. The overall incidence of candidemia at our center was 7.76 per 1000 admitted patients. In the ICU, the incidence was 14.95 per 1000 ICU admissions and the incidence in other wards was 4.09 per 1000 admissions. The central venous catheter was present in 119 (75.8\%) patients, whereas, the peripheral venous catheter was found inserted in 32 (20.4\%) patients, who developed candidemia. Total parenteral nutrition was administered to 37 (23.6\%) patients, who developed candidemia. All these patients who had candidemia received broad spectrum antibiotics.

Out of the 212 isolates, Candida tropicalis, 82 (38.7\%), was the most common, followed by C. parapsilosis, 43 (20.3\%), C. albicans, 29 (13.7\%), C. glabrata, 24 (11.3\%), C. rugosa, 20 (9.4\%), C. hemulonii, 6 (2.8\%), C. guilliermondii, 4 (1.8\%), C. famata, 3 (1.4\%), and C. lusitaniae, 1 (0.47\%) [Figure 1]. The major injuries of the patients and the species isolated from those patients are presented in Table 1.

During the study period, 2574 central venous catheter tips were sent to our laboratory for culture [Table 2]. Only nineteen patients had the same Candida isolate recovered from the central venous pressure (CVP) tips, (C. tropicalis (9), C. parapsilosis (4), C. albicans (3), and C. glabrata (3)), and therefore, had Central Line

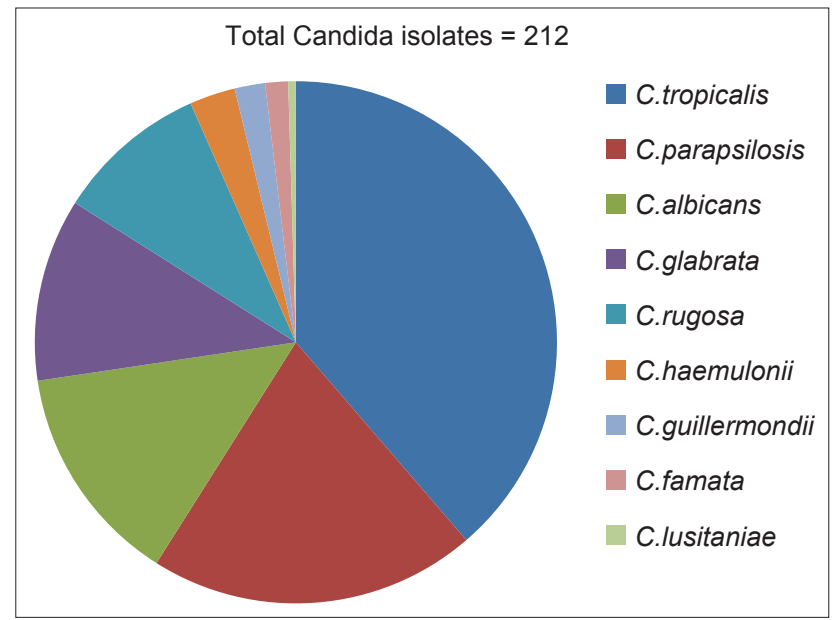

Figure 1: Distribution of Candida species
Associated Blood Stream Infections (CLABSI) due to Candida spp [Table 3]. As not all the central venous catheter tips were sent to the laboratory, due to the hemodynamic instability of the patient, the catheters were not changed, and even those catheters that were pulled out were often, but not always, sent to the laboratory. Many a times the CVP tips sent for culture showed growth of Candida spp. in the absence of a positive blood culture. These tips were colonized by Candida spp without being spread in the systemic blood circulation. On account of these constraints, it was extremely difficult to correctly identify the primary CLABSI from the non-CLABSI cases, due to secondary candidemia.

Candidemia developed in patients after an average of 15 days of intra-hospital stay (range 1 to 103 days). Antifungal treatment was given to 93 patients. Among them there were four patients who expired, despite having started the antifungal treatment, but the treatment was effective in the other 89 patients. Prophylactic antifungal therapy was not a routine practice at our institute. Restricted use of antifungals was practiced and antifungal therapy was initiated on evidence of positive fungal culture reports. However, five patients with abdominal injury, who had undergone gastrointestinal (GI) surgery and were on total parenteral nutrition and suffering from high-grade fever, with negative bacterial blood cultures, were empirically started on fluconazole.

Mortality in trauma patients occurs due to multiple factors like severity of injury, age of the patient, underlying comorbid conditions, time elapsed before treatment was started, initial immune activation followed by immunosuppression due to trauma, concomitant infections like blood stream infection, ventilator-associated pneumonia, and other bacterial infections, besides candidemia, prompt administration of appropriate antibiotic or antifungal therapy. Therefore, only an attributable mortality can be determined if we compare these statistics with other suitable controls in a case-control study. As our study was not a case-control study only crude mortality could be determined. Of the 157 patients who had candidemia, $68(43.31 \%)$ had a fatal outcome. The median duration of survival after development of candidemia was 10 days, with a range of 0 to 132 days.

\begin{tabular}{|c|c|c|c|c|c|c|c|c|c|}
\hline Injury (no. of isolates) & C.albicans & C.tropicalis & C.parapsilosis & C.rugosa & C.glabrata & C.guilliermondii & C.famata & C.haemulonii & C.lusitaniae \\
\hline Head (121) & 9 & 51 & 24 & 15 & 19 & - & 1 & 2 & - \\
\hline Spine (28) & 6 & 6 & 7 & 2 & - & 4 & 1 & 1 & 1 \\
\hline Abdomen (32) & 4 & 15 & 7 & - & 4 & - & & 2 & \\
\hline Chest (5) & - & 1 & 3 & - & - & - & - & 1 & - \\
\hline Orthopedic+Polytrauma (26) & 10 & 9 & 2 & 3 & 1 & - & 1 & - & - \\
\hline
\end{tabular}


The majority of patients who had a fatal outcome had C. tropicalis infection in 29 (43\%), followed by C. parapsilosis in $15(22 \%)$, C. albicans in 9 (13\%), C. glabrata in $7(10 \%)$, C. rugosa in $6(9 \%)$, and C. guilliermondii in $2(3 \%)$.

Resistance to antifungal agents was observed in a few isolates. A total of seven (3.3\%) (C. glabrata 6, C. rugosa 1 ) isolates were resistant to fluconazole and six $(2.8 \%)$ were intermediately sensitive to fluconazole. A total of seven $(3.3 \%)$ (C. glabrata 6 C. albicans 1$)$ isolates were resistant to Amphotericin B and three (1.4\%) were intermediately sensitive to Amphotericin B. Six (20\%) isolates of C. glabrata were resistant to both fluconazole and Amphotericin B. No resistance was seen for flucytosine or voriconazole.

\section{DISCUSSION}

Trauma is one of the most common global causes of mortality in young adults. Infections are the leading cause of death in trauma victims. Very few studies have been done on the profile of candidemia in this population. Knowledge of the epidemiology of candidemia can help to salvage these patients, who are in the economically productive age group, with few, if any, underlying medical conditions.

\begin{tabular}{lc}
\hline Table 2: Results of Central venous catheter \\
tips cultured from January & $\mathbf{2 0 0 9}$ to \\
July & $\mathbf{2 0 1 2}$ \\
\hline Organism grown & No. of CVP tips \\
\hline Sterile & 785 \\
Mixture of bacteria & 132 \\
Diptheroids & 135 \\
Acinetobacter spp. & 56 \\
Klebsiella spp. & 44 \\
Pseudomonas spp. & 17 \\
Candida spp. & 24 \\
Staphylococcus aureus & 32 \\
Staphylococcus hemolyticus & 27 \\
Staphylococcus epidermidis & 11 \\
Staphylococcus hominis & 4 \\
Staphylococcus cohnii & 1 \\
Stenotrophomans maltophila & 6 \\
Serratia marsecens & 4 \\
Enterobacter spp. & 9 \\
Enterococcus spp. & 2 \\
Providencia spp. & 1 \\
Burkholderia cepacia & 1 \\
Total & 2574 \\
\hline
\end{tabular}

Candidemia is an emerging problem in healthcare settings. In a ten-year study at a tertiary care hospital in Canada, Taylor et al. reported an increase in the cases of candidemia from 4.8/1000 admissions in 1986 to 11.4/1000 admissions in 1996. ${ }^{[16]}$ Pfaller et al. have also reported an increase in invasive candidiasis rates from 19 to 20 per 10,000 hospital discharges in 1996 to 24 per 10,000 hospital discharges in $2003 .{ }^{[13]}$ The incidence of candidemia varies from $0.77-77$ per 1000 admissions/discharges. ${ }^{[17,18]}$ The overall incidence of candidemia at our center is 7.76 per 1000 admitted patients, which is in correlation with the findings of the studies conducted at various Indian hospitals, which report an incidence of 1 - 12 cases of candidemia per 1000 admissions. ${ }^{[19]}$

Various studies conducted at different hospitals around the globe have found a higher incidence of candidemia in ICUs as compared to general wards. A nation-wide study from Israel reported candidemia rates in 18 different Israeli hospitals, which ranged from 0.1 to $0.01 \%$ of all admissions, with a mean of $0.05 \%$. They also found that the incidence of candidemia differed significantly between the wards, which ranged from 4 - 5/10,000 in the General Surgery and Internal Medicine wards to about $60 / 10,000$ and $80 / 10,000$ in the Intensive Care and Preterm units, respectively. ${ }^{[20]}$ Marchetti et al., in their study over a period of 10 years in Swiss tertiary care hospitals, found that candidemia is five to ten times more common in ICUs as compared to other wards. ${ }^{[2]}$ Even at our center, the incidence of candidemia is almost four times more common in ICUs as compared to the wards. In the ICU, the incidence is 14.95 per 1000 ICU admissions and the incidence in other wards is 4.09 per 1000 admissions. Montagna et al. in their study covering 18 ICUs in southern Italy reported an incidence of 16.5 cases per 1000 admissions. ${ }^{[21]}$ Molina et al. have reported 34.3 episodes of candidemia per 1000 ICU patients. ${ }^{[2]}$ An Indian neonatal ICU has reported an incidence of 77 cases per 1000 discharges in that hospital. ${ }^{[18]}$ Frausto $e t$ al. in an 18-month study conducted at seven surgical ICUs (SICUs) and six neonatal ICUs (NICUs) at six different hospitals of USA found an incidence of 9.8/1000 admissions in Surgical Intensive Care Units (SICUs) and 12.3/1000 admissions in the Neonatal Intensive Care Units (NICUs). ${ }^{[23]}$

Recent studies have shown an increase in the incidence of candidemia due to non-albicans Candida, with the isolation

\begin{tabular}{lccccccc}
\hline \multicolumn{6}{l}{ Table 3: Results of CVP tips growing } & Candida spp. in CLABSI and Non-CLABSI patients \\
\hline Patient group & Total number of CVP tips & C.albicans & C.tropicalis & C.parapsilosis & C.rugosa & C.glabrata & C.guillermondii \\
\hline CLABSI & 19 & 3 & 9 & 4 & - & 3 & - \\
Non- CLABSI/Colonizers & 5 & 4 & 1 & - & - & - & - \\
\hline
\end{tabular}

CVP: Central venous pressure, CLABSI: Central line associated bloodstream infection 
rate ranging from 50 to $96 \%$ from tertiary care centers in India. ${ }^{[24-27]}$ Non-albicans Candida caused about $86 \%$ cases of candidemia in our study. C. tropicalis and C. parapsilosis are an emerging cause of candidemia in India. In our study, $59 \%$ of all candidemia cases were caused by these two species. Previous studies by Chakrabarti et al., ${ }^{[25]}$ Xess et al. ${ }^{[26]}$ and Singh et al. ${ }^{[27]}$ are in agreement with our study, and it appears that $C$. tropicalis is the predominant species causing candidemia in North India. Wormwood et al. ${ }^{[28]}$ also found $C$. tropicalis as the predominant species causing candidemia in Chile.

There were multiple risk factors for these critically injured trauma patients. All of them received broad spectrum antibiotics, had central and peripheral lines, and due to the nature of their injury had a prolonged hospital stay. The odds of developing candidemia in critical patients, who are being invasively monitored and receiving broad spectrum antibiotics increases as the duration of hospital stay increases. Wolf et al. ${ }^{[29]}$ reported that candidemia developed after 12 days of injury (4 - 16 days). Wormwood et al. ${ }^{[28]}$ found that patients had an intrahospital stay of 11 days (0 - 36 days) before developing invasive candidiasis. Bougnoux et al..$^{[30]}$ observed that candidemia developed after a mean of $19+2.9$ days of ICU admission. In our study, on an average, candidemia developed in these patients, 15 days after sustaining the injury.

The crude mortality for candidemia ranged from 30 - 81\%. In our study, mortality was seen in $43 \%$ of these patients, which was in concordance with the studies of Bassetti et al. ${ }^{[31]}(43.5 \%)$, Singh et al. ${ }^{[27]}(50.6 \%)$, Chakrabarti et al. ${ }^{[25]}(54.3 \%)$, and Bougnoux et al. ${ }^{[30]}(61.8 \%)$. In our study, C. tropicalis (43\%) was the predominant species isolated from the patients who died. This was probably a reflection of the most prevalent species in our institute and did not necessarily indicate any enhanced virulence potential.

About $3.3 \%$ of the strains were resistant to fluconazole in our study. The prevalence of resistance in our study was less when compared with previous studies by Singh et al. ${ }^{[2]}(6.7 \%)$, Chakrabarti et al. ${ }^{[25]}(7.1 \%)$, Wormwood et al. ${ }^{[28]}(11.2 \%)$, and Bassetti et al. ${ }^{[31]}(12.6 \%)$. We also found resistance to Amphotericin B in 3.3\% of the isolates. Previous studies by Singh et al. ${ }^{[27]}$ and Xess et al. ${ }^{\left[{ }^{[2]}\right.}$ did not find any resistance to amphotericin B. Chakrabarti et al., ${ }^{[24]}$ however, had reported resistance to Amphotericin B in C. albicans (15.4\%), C. tropicalis (8.1\%), and C. krusei (33.3\%) strains. Therefore, emergence of resistance to the polyene group of antifungals is a matter of grave concern and will require careful and close monitoring in future.

\section{CONCLUSION}

To conclude, candidemia is a significant cause of mortality in trauma patients in our center, with C. tropicalis and C. parapsilosis being the predominant pathogens. Resistance to antifungals is gradually increasing, and thus, there is an emergent need for antibiotic stewardship and maintenance of aseptic techniques, while inserting and maintaining invasive devices, frequent clinical checks for weaning these patients off these invasive monitoring devices, and better hand hygiene practices.

\section{REFERENCES}

1. Wisplinghoff H, Bischoff T, Tallent SM, Seifert H, Wenzel RP, Edmond MB. Nosocomial bloodstream infections in US hospitals: Analysis of 24,179 cases from a prospective nationwide surveillance study. Clin Infect Dis 2004;39:309-17.

2. Marchetti O, Bille J, Fluckiger U, Eggimann P, Ruef C, Garbino J, et al. Epidemiology of candidemia in Swiss tertiary care hospitals: Secular trends 1991-2000. Clin Infect Dis 2004;38:311-20.

3. Zaoutis TE, Argon J, Chu J, Berlin JA, Walsh TJ, Feudtner C. The epidemiology and attributable outcomes of candidemia in adults and children hospitalized in the United States: A propensity analysis. Clin Infect Dis 2005;41:1232-9.

4. Horn DL, Neofytos D, Anaissie EJ, Fishman JA, Steinbach WJ, Olyaei AJ, et al. Epidemiology and outcomes of candidemia in 2019 patients: Data from the prospective antifungal therapy alliance registry. Clin Infect Dis 2009;48:1695-703.

5. Gudlaugsson O, Gillespie S, Lee K, Vande BJ, Hu J, Messer S, et al. Attributable mortality of nosocomial candidemia, revisited. Clin Infect Dis 2003;37:1172-7.

6. Rentz AM, Halpern MT, Bowden R. The impact of candidemia on length of hospital stay, outcome, and overall cost of illness. Clin Infect Dis 1998;27:781-8

7. Wey SB, Mori M, Pfaller MA, Woolson RF, Wenzel RP. Hospital acquired candidemia. The attributable mortality and excess length of stay. Arch Intern Med 1988;148:2642-5.

8. Fraser VJ, Jones M, Dunkel J, Storfer S, Medoff G, Dunagan WC. Candidemia in a tertiary care hospital: Epidemiology, risk factors, and predictors of mortality. Clin Infect Dis 1992;15:414-21.

9. Nguyen MH, Peacock JE, Tanner DC, Morris AJ, Nguyen ML, Snydman DR, et al. Therapeutic approaches in patients with candidemia. Evaluation in a multicenter, prospective, observational study. Arch Intern Med 1995;155:2429-35.

10. Nucci M, Colombo AL, Silveira F, Richtmann R, Salomao R, Branchini ML, et al. Risk factors for death in patients with candidemia. Infect Control Hosp Epidemiol 1998;19:846-50.

11. Mean M, Marchetti O, Calandra T. Bench-to-bedside review: Candida infections in the intensive care unit. Crit Care 2008;12:204-12.

12. Bouza E, Munoz P. Epidemiology of candidemia in intensive care units. Int J Antimicrob Agents 2008;32 Suppl 2:S87-91.

13. Pfaller MA, Diekema DJ. Epidemiology of Invasive Candidiasis: A Persistent Public Health Problem. Clin Microbiol Rev 2007;20:133-63.

14. Sobel JD. The emergence of non-albicans Candida species as causes of invasive candidiasis and candidemia. Curr Infect Dis Rep 2006;8:427-33.

15. Washington WC, Koneman EW, Stephen DA, Gary WP, Paul SC, William JM, et al. Koneman's Colour Atlas and Textbook of Diagnostic Microbiology. $6^{\text {th }}$ ed. Philadelphia: Lippincott Williams and Wilkins; 2006.

16. Taylor G, Buchanan-Chell M, Kirkland T, McKenzie M, Wiens R. Long term trends in the occurrence of nosocomial blood stream infection. Can J Infect Dis 2000;11:29-33. 
17. Ellis M, Hedstrom U, Jumaa P, Bener A. Epidemiology, presentation, management and outcome of candidemia in a tertiary care teaching hospital in the United Arab Emirates, 1995-2001. Med Mycol 2003;41:521-8.

18. Narang A, Agarwal PB, Chakrabarti A, Kumar P. Epidemiology of systemic candidiasis at a tertiary care neonatal unit. J Trop Pediatr 1998;44:104-8.

19. Chakrabarti A, Chatterjee SS, Shivaprakash MR. Overview of opportunistic fungal infections in India. Jpn J Med Mycol 2008;49:165-72.

20. Rennert G, Rennert HS, Pitlik S, Finkelstein R, Kitzes-Cohen R. Epidemiology of candidemia-A nationwide survey in Israel. Infection 2000;28:26-9.

21. Montagna MT, Caggiano G, Lovero G, De Giglio O, Coretti C, Cuna T, et al. Epidemiology of invasive fungal infections in the intensive care unit: Results of a multicenter Italian survey (AURORA Project). Infection 2013;41:645-53.

22. Gonzalez de Molina FJ, Leon C, Ruiz-Santana S, Saavedra P. CAVA I Study Group. Assessment of candidemia-attributable mortality in critically ill patients using propensity score matching analysis. Crit Care 2012;16:R105.

23. Rangel-Frausto MS, Wiblin T, Blumberg HM, Saiman L, Patterson J, Michael Rinaldi, et al. National Epidemiology of Mycoses Survey (NEMIS): Variations in Rates of Bloodstream Infections Due to Candida Species in Seven Surgical Intensive Care Units and Six Neonatal Intensive Care Units. Clin Infect Dis 1999;29:253-8.

24. Chakrabarti A, Mohan B, Shrivastava SK, Marak RS, Ghosh A, Ray P. Change in distribution and antifungal susceptibility of Candida species isolated from candidaemia cases in a tertiary care center during 1996-2000. Indian J Med Res 2002;116:5-12.

25. Chakrabarti A, Chatterjee SS, Rao KL, Zameer MM, Shivaprakash MR, Singhi S, et al. Recent experience with fungaemia: Change in species distribution and azole resistance. Scand J Infect Dis 2009;41:275-84.

26. Xess I, Jain N, Hasan F, Mandal P, Banerjee U. Epidemiology of candidemia in a tertiary care center of North India: 5 year study. Infection 2007;35:256-9.

27. Singh RI, Xess I, Mathur P, Behera B, Gupta B, Misra MC. Epidemiology of candidemia in critically ill trauma patients: Experiences of a level I trauma center in North India. J Med Microbiol 2011;60:342-8.

28. Ajenjo HM, Aquevedo SA, Guzmán DA, Poggi MH, Calvo AM, Castillo VC, et al. Epidemiological profile of invasive candidiasis in critically ill patients units at a University Hospital. Rev Child Infect 2011;28:118-22.

29. Wolf DG, Polacheck I, Block C, Sprung CL, Muggia-Sullam M, Wolf YG, et al. High rate of Candidemia in patients sustaining injuries in a bomb blast at a marketplace: A possible environmental source. Clin Infect Dis 2000;31:712-6.

30. Bougnoux ME, Kac G, Aegerter P, d'Enfert C, Fagon JY; CandiRea Study Group. Candidemia and candiduria in critically ill patients admitted to intensive care units in France: Incidence, molecular diversity, management and outcome. Intensive Care Med 2008;34:292-9.

31. Bassetti M, Taramasso L, Nicco E, Molinari MP, Mussap M, Viscoli C. Epidemiology, species distribution, antifungal susceptibility and outcome of nosocomial candidemia in a tertiary care hospital in Italy. PLoS One 2011;6:e24198.

How to cite this article: Tak V, Mathur P, Varghese P, Gunjiyal J, Xess I, Misra MC. The epidemiological profile of candidemia at an Indian trauma care center. J Lab Physicians 2014;6:96-101.

Source of Support: Nil. Conflict of Interest: None declared. 